\title{
Marcinkiewicz Integral Operators and Commutators on Herz Spaces with Variable Exponents
}

\author{
Liwei Wang \\ School of Mathematics and Physics, Anhui Polytechnic University, Wuhu 241000, China \\ Correspondence should be addressed to Liwei Wang; wangliwei8013@163.com
}

Received 26 July 2014; Accepted 21 September 2014; Published 15 October 2014

Academic Editor: Dashan Fan

Copyright (C) 2014 Liwei Wang. This is an open access article distributed under the Creative Commons Attribution License, which permits unrestricted use, distribution, and reproduction in any medium, provided the original work is properly cited.

Our aim in this paper is to give the boundedness of the Marcinkiewicz integral $\mu_{\Omega}$ on Herz spaces $\dot{K}_{p(\cdot)}^{\alpha(\cdot), q}\left(\mathbb{R}^{n}\right)$ and $K_{p(\cdot)}^{\alpha(\cdot),}\left(\mathbb{R}^{n}\right)$, where the two main indices are variable. Meanwhile, we consider the boundedness of the higher order commutator $\mu_{\Omega b}^{m}$ generated by $\mu_{\Omega}$ and a function $b$ in $\mathrm{BMO}\left(\mathbb{R}^{n}\right)$ on these spaces.

\section{Introduction}

Let $\mathbb{S}^{n-1}$ be the unit sphere in $\mathbb{R}^{n}(n \geq 2)$ equipped with the normalized Lebesgue measure $d \sigma\left(x^{\prime}\right)$. Suppose that $\Omega$ is homogeneous of degree zero on $\mathbb{R}^{n}$ and has mean zero on $\mathbb{S}^{n-1}$, that is,

$$
\int_{\mathbb{S}^{n-1}} \Omega\left(x^{\prime}\right) d \sigma\left(x^{\prime}\right)=0 .
$$

Then the Marcinkiewicz integral $\mu_{\Omega}$ in higher dimension is defined by

$$
\mu_{\Omega}(f)(x)=\left(\int_{0}^{\infty}\left|F_{\Omega, t}(f)(x)\right|^{2} \frac{d t}{t^{3}}\right)^{1 / 2}
$$

where

$$
F_{\Omega, t}(f)(x)=\int_{|x-y| \leq t} \frac{\Omega(x-y)}{|x-y|^{n-1}} f(y) d y .
$$

Denote by $\mathbb{N}$ the set of all positive integer numbers. Let $m \in \mathbb{N}$ and $b \in \operatorname{BMO}\left(\mathbb{R}^{n}\right)$; the higher order commutator $\mu_{\Omega, b}^{m}$ is defined by

$$
\mu_{\Omega, b}^{m}(f)(x)=\left(\int_{0}^{\infty}\left|F_{\Omega, b, t}^{m}(f)(x)\right|^{2} \frac{d t}{t^{3}}\right)^{1 / 2},
$$

where

$$
F_{\Omega, b, t}^{m}(f)(x)=\int_{|x-y| \leq t} \frac{\Omega(x-y)}{|x-y|^{n-1}}(b(x)-b(y))^{m} f(y) d y .
$$

Stein [1] defined the operator $\mu_{\Omega}$ and proved that if $\Omega \epsilon$ $\operatorname{Lip}_{\gamma}\left(\mathbb{S}^{n-1}\right)(0<\gamma \leq 1)$, then $\mu_{\Omega}$ is of type $(p, p)(1<p \leq 2)$ and of weak type $(1,1)$. Benedek et al. [2] showed that $\mu_{\Omega}$ is of type $(p, p)(1<p<\infty)$ with $\Omega \in C^{1}\left(\mathbb{S}^{n-1}\right)$. Ding et al. [3] improved the previous results to the case of $\Omega \in H^{1}\left(\mathbb{S}^{n-1}\right)$, where $H^{1}\left(\mathbb{S}^{n-1}\right)$ denotes the Hardy space on $\mathbb{S}^{n-1}$. Obviously, $\mu_{\Omega, b}^{1}=\left[b, \mu_{\Omega}\right]$, which was defined by Torchinsky and Wang in [4]; moreover, they proved that if $\Omega \in \operatorname{Lip}_{\gamma}\left(\mathbb{S}^{n-1}\right)(0<$ $\gamma \leq 1)$, then $\left[b, \mu_{\Omega}\right]$ is bounded on $L^{p}\left(\mathbb{R}^{n}\right)(1<p<\infty)$. Ding et al. [5] weakened the smoothness of the kernel to a rough kernel and showed that if $\Omega \in L^{q}\left(\mathbb{S}^{n-1}\right)(1<q \leq \infty)$, then $\left[b, \mu_{\Omega}\right]$ is of type $(p, p)(1<p<\infty)$. Ding et al. [6] established the weighted weak $L \log L$ type estimates for $\mu_{\Omega, b}^{m}$ when $\Omega \in \operatorname{Lip}_{\gamma}\left(\mathbb{S}^{n-1}\right)(0<\gamma \leq 1)$. Recently, Zhang [7] improved the previous result and proved that $\mu_{\Omega, b}^{m}$ enjoys the same weighted weak $L \log L$ type estimates when the kernel $\Omega$ satisfies a kind of Dini's conditions. For further details on recent developments on this field, we refer the readers to $[8,9]$ and references therein.

Function spaces with variable exponents were intensively studied during the past 20 years, due to their applications to PDE with nonstandard growth conditions and so on; we mention $[10,11]$, for instance. Since the fundamental paper [12] by Kováćik and Rákosník appeared in 1991, the Lebesgue spaces with variable exponent $L^{p(\cdot)}\left(\mathbb{R}^{n}\right)$ have attracted a great attention and many interesting results have been obtained; 
see [13-15]. Izuki [16, 17] defined the Herz spaces $\dot{K}_{p(\cdot)}^{\alpha, q}\left(\mathbb{R}^{n}\right)$ and $K_{p(\cdot)}^{\alpha, q}\left(\mathbb{R}^{n}\right)$ with variable exponent $p$ but fixed $\alpha \in \mathbb{R}$ and $q \in(0, \infty]$. Wang et al. $[18,19]$ obtained the boundedness of $\mu_{\Omega}$ and $\left[b, \mu_{\Omega}\right]$ on $\dot{K}_{p(\cdot)}^{\alpha, q}\left(\mathbb{R}^{n}\right)$ and $K_{p(\cdot)}^{\alpha, q}\left(\mathbb{R}^{n}\right)$. Almeida and Drihem [20] established the boundedness of a wide class of sublinear operators, which includes maximal, potential, and Calderón-Zygmund operators, on Herz spaces $\dot{K}_{p(\cdot)}^{\alpha(\cdot), q}\left(\mathbb{R}^{n}\right)$ and $K_{p(\cdot)}^{\alpha(\cdot), q}\left(\mathbb{R}^{n}\right)$, where the two main exponents $\alpha$ and $p$ are both variable. In this paper we will give boundedness results for $\mu_{\Omega}$ and $\mu_{\Omega, b}^{m}$ on Herz spaces $\dot{K}_{p(\cdot)}^{\alpha(\cdot), q}\left(\mathbb{R}^{n}\right)$ and $K_{p(\cdot)}^{\alpha(\cdot), q}\left(\mathbb{R}^{n}\right)$.

For brevity, $|E|$ denotes the Lebesgue measure for a measurable set $E \subset \mathbb{R}^{n} . f_{E}$ denotes the integral average of $f$ on $E$, that is, $f_{E}=|E|^{-1} \int_{E} f(x) d x \cdot p^{\prime}(\cdot)$ stands for the conjugate exponent $1 / p(\cdot)+1 / p^{\prime}(\cdot)=1 . B(x, r)=\{y \epsilon$ $\left.\mathbb{R}^{n}:|x-y|<r\right\}$. $C$ denotes a positive constant, which may have different values even in the same line. $f \leqslant g$ means that $f \leq C g$, and $f \approx g$ means that $f \leqslant g \leqslant f$.

\section{Preliminaries and Main Results}

Let $E \subset \mathbb{R}^{n}$ with $|E|>0$, and let $p(\cdot): E \rightarrow[1, \infty)$ be a measurable function. Let us first recall some definitions and notations.

Definition 1. The Lebesgue space with variable exponent $L^{p(\cdot)}(E)$ is defined by

$$
\begin{aligned}
& L^{p(\cdot)}(E) \\
& =\left\{f \text { is measurable : } \int_{E}\left(\frac{|f(x)|}{\lambda}\right)^{p(x)} d x<\infty\right. \\
& \text { for some constant } \lambda>0\} .
\end{aligned}
$$

This is a Banach space with the Luxemburg norm

$$
\|f\|_{L^{p(\cdot)}(E)}=\inf \left\{\lambda>0: \int_{E}\left(\frac{|f(x)|}{\lambda}\right)^{p(x)} d x \leq 1\right\} .
$$

Let $f \in L_{\text {loc }}^{1}(E)$; the Hardy-Littlewood maximal operator $M$ is defined by

$$
M f(x)=\sup _{r>0} r^{-n} \int_{B(x, r) \cap E}|f(y)| d y .
$$

Denote

$$
\begin{gathered}
p_{-}=\operatorname{ess} \inf \{p(x): x \in E\}, \\
p_{+}=\operatorname{ess} \sup \{p(x): x \in E\}, \\
\mathscr{P}(E)=\left\{p(\cdot): p_{-}>1, p_{+}<\infty\right\}, \\
\mathscr{B}(E)=\left\{p(\cdot) \in \mathscr{P}(E): M \text { is bounded on } L^{p(\cdot)}(E)\right\} .
\end{gathered}
$$

Let $B_{k}=\left\{x \in \mathbb{R}^{n}:|x| \leq 2^{k}\right\}, R_{k}=B_{k} \backslash B_{k-1}$, and $\chi_{k}=\chi_{R_{k}}$ be the characteristic function of the set $R_{k}$ for $k \in \mathbb{Z}$. For $m \in$ $\mathbb{N}$, one denotes $\widetilde{\chi_{m}}=\chi_{R_{m}}$ if $m \geq 1$, and $\widetilde{\chi_{0}}=\chi_{B_{0}}$. By $\ell^{q}(0<$ $q \leq \infty)$, we denote the discrete Lebesgue space equipped by the usual quasinorm.

Definition 2. Let $0<q \leq \infty, p(\cdot) \in \mathscr{P}\left(\mathbb{R}^{n}\right)$, and $\alpha(\cdot): \mathbb{R}^{n} \rightarrow$ $\mathbb{R}$ with $\alpha \in L^{\infty}\left(\mathbb{R}^{n}\right)$.

(1) The homogeneous Herz space $\dot{K}_{p(\cdot)}^{\alpha(\cdot), q}\left(\mathbb{R}^{n}\right)$ is defined by

$$
\dot{K}_{p(\cdot)}^{\alpha(\cdot), q}\left(\mathbb{R}^{n}\right)=\left\{f \in L_{\text {loc }}^{p(\cdot)}\left(\mathbb{R}^{n} \backslash\{0\}\right):\|f\|_{\dot{K}_{p(\cdot)}^{\alpha(\cdot),}\left(\mathbb{R}^{n}\right)}<\infty\right\},
$$

where

$$
\|f\|_{\dot{K}_{p(\cdot)}^{\alpha(\cdot), q}\left(\mathbb{R}^{n}\right)}=\left\|\left\{\left\|2^{\alpha(\cdot) k} f \chi_{k}\right\|_{L^{p(\cdot)}\left(\mathbb{R}^{n}\right)}\right\}_{k=-\infty}^{\infty}\right\|_{\ell^{q}(\mathbb{Z})} .
$$

(2) The inhomogeneous Herz space $K_{p(\cdot)}^{\alpha(\cdot), q}\left(\mathbb{R}^{n}\right)$ is defined by

$$
K_{p(\cdot)}^{\alpha(\cdot), q}\left(\mathbb{R}^{n}\right)=\left\{f \in L_{\text {loc }}^{p(\cdot)}\left(\mathbb{R}^{n}\right):\|f\|_{K_{p(\cdot)}^{\alpha(\cdot), q}\left(\mathbb{R}^{n}\right)}<\infty\right\},
$$

where

$$
\|f\|_{K_{p(\cdot)}^{\alpha(\cdot), q}\left(\mathbb{R}^{n)}\right.}=\left\|\left\{\left\|2^{\alpha(\cdot) m} f \widetilde{\chi_{m}}\right\|_{L^{p^{(\cdot)}\left(\mathbb{R}^{n}\right)}}\right\}_{m=0}^{\infty}\right\|_{\mathcal{R}^{q}(\mathbb{N})},
$$

with the usual modification when $q=\infty$.

Remark 3. It is obvious that if $0<q_{1} \leq q_{2} \leq \infty$, then $\dot{K}_{p(\cdot)}^{\alpha(\cdot), q_{1}}\left(\mathbb{R}^{n}\right) \subset \dot{K}_{p(\cdot)}^{\alpha(\cdot), q_{2}}\left(\mathbb{R}^{n}\right)$ and $K_{p(\cdot)}^{\alpha(\cdot), q_{1}}\left(\mathbb{R}^{n}\right) \subset K_{p(\cdot)}^{\alpha(\cdot), q_{2}}\left(\mathbb{R}^{n}\right)$. If both $\alpha(\cdot)$ and $p(\cdot)$ are constants, then $\dot{K}_{p(\cdot)}^{\alpha(\cdot), q}\left(\mathbb{R}^{n}\right)=\dot{K}_{p}^{\alpha, q}\left(\mathbb{R}^{n}\right)$ and $K_{p(\cdot)}^{\alpha(\cdot), q}\left(\mathbb{R}^{n}\right)=K_{p}^{\alpha, q}\left(\mathbb{R}^{n}\right)$ are classical Herz spaces; see $[21,22]$.

Definition 4. A function $\alpha(\cdot): \mathbb{R}^{n} \rightarrow \mathbb{R}$ is called log-Hölder continuous at the origin, if there exists a constant $C_{\log }>0$ such that

$$
|\alpha(x)-\alpha(0)| \leq \frac{C_{\log }}{\log (e+1 /|x|)},
$$

for all $x \in \mathbb{R}^{n}$. If, for some $\alpha_{\infty} \in \mathbb{R}$ and $C_{\log }>0$, there holds

$$
\left|\alpha(x)-\alpha_{\infty}\right| \leq \frac{C_{\log }}{\log (e+|x|)}
$$

for all $x \in \mathbb{R}^{n}$, then $\alpha(\cdot)$ is called log-Hölder continuous at infinity.

Let one denote

$$
\begin{aligned}
& \left\|\left\{h_{k}\right\}\right\|_{\ell_{>}^{q}\left(L^{p(\cdot)}\right)}=\left(\sum_{k \geqslant 0}\left\|h_{k}\right\|_{L^{p(\cdot)}}^{q}\right)^{1 / q}, \\
& \left\|\left\{h_{k}\right\}\right\|_{\ell_{<}^{q}\left(L^{p(\cdot)}\right)}=\left(\sum_{k<0}\left\|h_{k}\right\|_{L^{p(\cdot)}}^{q}\right)^{1 / q}
\end{aligned}
$$


for sequences $\left\{h_{k}\right\}_{k \in \mathbb{Z}}$ of measurable functions (with the usual modification when $q=\infty$ ).

Proposition 5 (see [20]). Let $0<q \leq \infty, p(\cdot) \in \mathscr{P}\left(\mathbb{R}^{n}\right)$, and $\alpha(\cdot) \in L^{\infty}\left(\mathbb{R}^{n}\right)$. If $\alpha(\cdot)$ is log-Hölder continuous both at the origin and at infinity, then

$$
\begin{aligned}
\|f\|_{\dot{K}_{p(\cdot)}^{\alpha(\cdot),}\left(\mathbb{R}^{n}\right)} \approx & \left\|\left\{2^{\alpha(0) k} f \chi_{k}\right\}\right\|_{\ell_{<}^{q}\left(L^{p(\cdot)}\right)} \\
& +\left\|\left\{2^{\alpha \infty^{k}} f \chi_{k}\right\}\right\|_{\ell_{>}^{q}\left(L^{p(\cdot)}\right)} .
\end{aligned}
$$

Before stating the main results of this paper, we introduce some key lemmas that will be used later.

Lemma 6 (generalized Hölder's inequality [12]). Let $p(\cdot) \in$ $\mathscr{P}\left(\mathbb{R}^{n}\right)$; if $f \in L^{p(\cdot)}\left(\mathbb{R}^{n}\right)$ and $g \in L^{p^{\prime}(\cdot)}\left(\mathbb{R}^{n}\right)$, then

$$
\int_{\mathbb{R}^{n}}|f(x) g(x)| d x \leq r_{p}\|f\|_{L^{p(\cdot)}\left(\mathbb{R}^{n}\right)}\|g\|_{L^{p^{\prime}(\cdot)\left(\mathbb{R}^{n}\right)}},
$$

where $r_{p}=1+1 / p_{-}-1 / p_{+}$.

We remark that the following Lemmas 7-9 were shown in Izuki $[17,23]$, and Lemma 10 was considered by Wang et al. in [18].

Lemma 7. Let $p(\cdot) \in \mathscr{B}\left(\mathbb{R}^{n}\right)$; then one has, for all balls $B$ in $\mathbb{R}^{n}$,

$$
\frac{1}{|B|}\left\|\chi_{B}\right\|_{L^{p(\cdot)}\left(\mathbb{R}^{n}\right)}\left\|\chi_{B}\right\|_{L^{p^{\prime} \cdot\left(\mathbb{R}^{n}\right)}} \lesssim 1 .
$$

Lemma 8. Let $p(\cdot) \in \mathscr{B}\left(\mathbb{R}^{n}\right)$; then one has, for all balls $B$ in $\mathbb{R}^{n}$ and all measurable subsets $S \subset B$,

$$
\frac{\left\|\chi_{S}\right\|_{L^{p(\cdot)}\left(\mathbb{R}^{n}\right)}}{\left\|\chi_{B}\right\|_{L^{p(\cdot)}\left(\mathbb{R}^{n}\right)}} \leqslant\left(\frac{|S|}{|B|}\right)^{\delta_{1}}, \quad \frac{\left\|\chi_{S}\right\|_{L^{p^{\prime}(\cdot)}\left(\mathbb{R}^{n}\right)}}{\left\|\chi_{B}\right\|_{L^{p^{\prime}(\cdot)}\left(\mathbb{R}^{n}\right)}} \lesssim\left(\frac{|S|}{|B|}\right)^{\delta_{2}},
$$

where $\delta_{1}$ and $\delta_{2}$ are constants with $0<\delta_{1}, \delta_{2}<1$.

Lemma 9. Let $m \in \mathbb{N}, b \in B M O\left(\mathbb{R}^{n}\right)$, and $k>i(k, i \in \mathbb{N})$; then one has

$$
\begin{gathered}
\sup _{B \subset \mathbb{R}^{n}} \frac{1}{\left\|\chi_{B}\right\|_{L^{p(\cdot)}\left(\mathbb{R}^{n}\right)}}\left\|\left(b-b_{B}\right)^{m} \chi_{B}\right\|_{L^{p(\cdot)}\left(\mathbb{R}^{n}\right)} \approx\|b\|_{B M O}^{m}, \\
\left\|\left(b-b_{B_{i}}\right)^{m} \chi_{B_{k}}\right\|_{L^{p(\cdot)}\left(\mathbb{R}^{n}\right)} \leqslant(k-i)^{m}\|b\|_{B M O}^{m}\left\|\chi_{B_{k}}\right\|_{L^{p(\cdot)}\left(\mathbb{R}^{n}\right)^{(}}
\end{gathered}
$$

Lemma 10. Let $\Omega \in \operatorname{Lip}_{\gamma}\left(\mathbb{S}^{n-1}\right)(0<\gamma \leq 1), b \in B M O\left(\mathbb{R}^{n}\right)$, and $p(\cdot) \in \mathscr{B}\left(\mathbb{R}^{n}\right)$; then one has

$$
\begin{gathered}
\left\|\mu_{\Omega}(f)\right\|_{L^{p(\cdot)}\left(\mathbb{R}^{n}\right)} \lesssim\|f\|_{L^{p(\cdot)}\left(\mathbb{R}^{n}\right)^{\prime}} \\
\left\|\mu_{\Omega, b}^{m}(f)\right\|_{L^{p(\cdot)}\left(\mathbb{R}^{n}\right)} \lesssim\|b\|_{B M O}^{m}\|f\|_{L^{p(\cdot)}\left(\mathbb{R}^{n}\right)^{\cdot}}
\end{gathered}
$$

Our results in this paper can be stated as follows.

Theorem 11. Let $\Omega \in \operatorname{Lip}_{\gamma}\left(\mathbb{S}^{n-1}\right)(0<\gamma \leq 1), 0<q \leq \infty$, and $p(\cdot) \in \mathscr{B}\left(\mathbb{R}^{n}\right)$. And let $\alpha(\cdot) \in L^{\infty}\left(\mathbb{R}^{n}\right)$ be log-Hölder continuous both at the origin and at infinity, such that $-n \delta_{1}<$ $\alpha(0) \leq \alpha_{\infty}<n \delta_{2}$, where $0<\delta_{1}, \delta_{2}<1$ are the constants appearing in Lemma 8; then the operator $\mu_{\Omega}$ is bounded on $\dot{K}_{p(\cdot)}^{\alpha(\cdot), q}\left(\mathbb{R}^{n}\right)$ and $K_{p(\cdot)}^{\alpha(\cdot), q}\left(\mathbb{R}^{n}\right)$.

Theorem 12. Let $\Omega \in \operatorname{Lip}_{\gamma}\left(\mathbb{S}^{n-1}\right)(0<\gamma \leq 1), b \in$ $B M O\left(\mathbb{R}^{n}\right), 0<q \leq \infty$, and $p(\cdot) \in \mathscr{B}\left(\mathbb{R}^{n}\right)$. And let $\alpha(\cdot) \in$ $L^{\infty}\left(\mathbb{R}^{n}\right)$ be log-Hölder continuous both at the origin and at infinity, such that $-n \delta_{1}<\alpha(0) \leq \alpha_{\infty}<n \delta_{2}$, where $0<\delta_{1}$, $\delta_{2}<1$ are the constants appearing in Lemma 8; then the higher order commutator $\mu_{\Omega, b}^{m}$ is bounded on $\dot{K}_{p(\cdot)}^{\alpha(\cdot), q}\left(\mathbb{R}^{n}\right)$ and $K_{p(\cdot)}^{\alpha(\cdot), q}\left(\mathbb{R}^{n}\right)$.

Remark 13. If $\alpha(\cdot) \equiv \alpha$ is constant, then the statements corresponding to Theorems 11 and 12 can be found in $[19,24]$. We consider only $0<q<\infty$ in Section 3. The arguments are similar in the case $q=\infty$.

\section{Proofs of the Theorems}

In this section, we prove the boundedness of $\mu_{\Omega}$ and $\mu_{\Omega, b}^{m}$ on $\dot{K}_{p(\cdot)}^{\alpha(\cdot),}\left(\mathbb{R}^{n}\right)$ (the same arguments can be used in $\left.K_{p(\cdot)}^{\alpha(\cdot), q}\left(\mathbb{R}^{n}\right)\right)$; some of our decomposition techniques are similar to those used by Dong and $\mathrm{Xu}$ in [25].

Proof of Theorem 11. In view of Proposition 5, we have

$$
\begin{aligned}
\left\|\mu_{\Omega}(f)\right\|_{\dot{K}_{p(\cdot)}^{\left.\alpha(\cdot), \mathbb{R}^{n}\right)}} \approx & \left\|\left\{2^{\alpha(0) k} \mu_{\Omega}(f) \chi_{k}\right\}\right\|_{\ell_{<}^{q}\left(L^{p(\cdot)}\right)} \\
& +\left\|\left\{2^{\alpha_{\infty} k} \mu_{\Omega}(f) \chi_{k}\right\}\right\|_{\ell_{>}^{q}\left(L^{p(\cdot)}\right)} \\
= & I_{<}+I_{>} .
\end{aligned}
$$

Let $f \in \dot{K}_{p(\cdot)}^{\alpha(\cdot), q}\left(\mathbb{R}^{n}\right)$; write

$$
f(x)=\sum_{i=-\infty}^{\infty} f(x) \chi_{i}(x)=\sum_{i=-\infty}^{\infty} f_{i}(x)
$$

Minkowski's inequality implies that

$$
\begin{aligned}
I_{<}= & \left\{\sum_{k=-\infty}^{-1} 2^{\alpha(0) k q}\left\|\mu_{\Omega}(f) \chi_{k}\right\|_{L^{p(\cdot)}\left(\mathbb{R}^{n}\right)}^{q}\right\}^{1 / q} \\
\lesssim & \left\{\sum_{k=-\infty}^{-1} 2^{\alpha(0) k q}\left(\sum_{i=-\infty}^{k-2}\left\|\mu_{\Omega}\left(f_{i}\right) \chi_{k}\right\|_{L^{p(\cdot)}\left(\mathbb{R}^{n}\right)}\right)^{q}\right\}^{1 / q} \\
& +\left\{\sum_{k=-\infty}^{-1} 2^{\alpha(0) k q}\left(\sum_{i=k-1}^{k+1}\left\|\mu_{\Omega}\left(f_{i}\right) \chi_{k}\right\|_{L^{p(\cdot)}\left(\mathbb{R}^{n}\right)}\right)^{q}\right\}^{1 / q} \\
& +\left\{\sum_{k=-\infty}^{-1} 2^{\alpha(0) k q}\left(\sum_{i=k+2}^{\infty}\left\|\mu_{\Omega}\left(f_{i}\right) \chi_{k}\right\|_{L^{p(\cdot)}\left(\mathbb{R}^{n}\right)}\right)^{q}\right\}^{1 / q} \\
= & E_{<}+F_{<}+G_{<\cdot}
\end{aligned}
$$


4

Journal of Function Spaces

Similarly we obtain

$$
\begin{aligned}
I_{>}= & \left\{\sum_{k=0}^{\infty} 2^{\alpha_{\infty} k q}\left\|\mu_{\Omega}(f) \chi_{k}\right\|_{L^{p(\cdot)}\left(\mathbb{R}^{n}\right)}^{q}\right\}^{1 / q} \\
\lesssim & \left\{\sum_{k=0}^{\infty} 2^{\alpha_{\infty} k q}\left(\sum_{i=-\infty}^{k-2}\left\|\mu_{\Omega}\left(f_{i}\right) \chi_{k}\right\|_{L^{p(\cdot)}\left(\mathbb{R}^{n}\right)}\right)^{q}\right\}^{1 / q} \\
& +\left\{\sum_{k=0}^{\infty} 2^{\alpha_{\infty} k q}\left(\sum_{i=k-1}^{k+1}\left\|\mu_{\Omega}\left(f_{i}\right) \chi_{k}\right\|_{L^{p(\cdot)}\left(\mathbb{R}^{n}\right)}\right)^{q}\right\}^{1 / q} \\
& +\left\{\sum_{k=0}^{\infty} 2^{\alpha_{\infty} k q}\left(\sum_{i=k+2}^{\infty}\left\|\mu_{\Omega}\left(f_{i}\right) \chi_{k}\right\|_{L^{p(\cdot)}\left(\mathbb{R}^{n}\right)}\right)^{q}\right\}^{1 / q} \\
= & E_{>}+F_{>}+G_{>} .
\end{aligned}
$$

Thus we get

$$
\left\|\mu_{\Omega}(f)\right\|_{\dot{K}_{p(\cdot)}^{\alpha(\cdot), q}\left(\mathbb{R}^{n}\right)} \lesssim E+F+G
$$

where $E=E_{<}+E_{>}, F=F_{<}+F_{>}$, and $G=G_{<}+G_{>}$.

For $F$, Lemma 10 yields

$$
\begin{aligned}
F= & F_{<}+F_{>} \\
\lesssim & \left\{\sum_{k=-\infty}^{-1} 2^{\alpha(0) k q}\left\|f_{k}\right\|_{L^{p(\cdot)}\left(\mathbb{R}^{n}\right)}^{q}\right\}^{1 / q} \\
& +\left\{\sum_{k=0}^{\infty} 2^{\alpha_{\infty} k q}\left\|f_{k}\right\|_{L^{p(\cdot)}\left(\mathbb{R}^{n}\right)}^{q}\right\}^{1 / q} \\
\leq & \left\|\left\{2^{\alpha(0) k} f \chi_{k}\right\}\right\|_{\ell_{<}^{q}\left(L^{p(\cdot)}\right)}+\left\|\left\{2^{\alpha_{\infty} k} f \chi_{k}\right\}\right\|_{\ell_{>}^{q}\left(L^{p(\cdot)}\right)} \\
\approx & \|f\|_{\dot{K}_{p(\cdot)}^{\alpha(\cdot), q}\left(\mathbb{R}^{n}\right)^{\cdot}}
\end{aligned}
$$

Now we turn to estimate $E$. Observe that if $x \in R_{k}, y \in R_{i}$, and $i \leq k-2$, then $|x-y| \approx|x| \approx 2^{k}$ and

$$
\left|\frac{1}{|x-y|^{2}}-\frac{1}{|x|^{2}}\right| \lesssim \frac{|y|}{|x-y|^{3}} .
$$

Since $\Omega \in \operatorname{Lip}_{\gamma}\left(\mathbb{S}^{n-1}\right) \subset L^{\infty}\left(\mathbb{S}^{n-1}\right)$, by Minkowski's inequality and Lemma 6 , we have

$$
\begin{aligned}
& \left|\mu_{\Omega}\left(f_{i}\right)(x)\right| \\
& \leq\left(\int_{0}^{|x|}\left|\int_{|x-y| \leq t} \frac{\Omega(x-y)}{|x-y|^{n-1}} f_{i}(y) d y\right|^{2} \frac{d t}{t^{3}}\right)^{1 / 2} \\
& \quad+\left(\int_{|x|}^{\infty}\left|\int_{|x-y| \leq t} \frac{\Omega(x-y)}{|x-y|^{n-1}} f_{i}(y) d y\right|^{2} \frac{d t}{t^{3}}\right)^{1 / 2}
\end{aligned}
$$

$$
\begin{aligned}
\lesssim & \int_{R_{i}} \frac{\left|f_{i}(y)\right|}{|x-y|^{n-1}}\left(\int_{|x-y| \leq t,|x| \geq t} \frac{d t}{t^{3}}\right)^{1 / 2} d y \\
& +\int_{R_{i}} \frac{\left|f_{i}(y)\right|}{|x-y|^{n-1}}\left(\int_{|x|}^{\infty} \frac{d t}{t^{3}}\right)^{1 / 2} d y \\
\lesssim & \int_{R_{i}} \frac{\left|f_{i}(y)\right|}{|x-y|^{n-1}} \cdot \frac{|y|^{1 / 2}}{|x-y|^{3 / 2}} d y+\int_{R_{i}} \frac{\left|f_{i}(y)\right|}{|x-y|^{n-1}} \cdot \frac{1}{|x|} d y \\
\lesssim & 2^{(i-k) / 2} 2^{-k n}\left\|f_{i}\right\|_{L^{p(\cdot)}\left(\mathbb{R}^{n}\right)}\left\|\chi_{i}\right\|_{L^{p^{\prime}(\cdot)}\left(\mathbb{R}^{n}\right)} \\
& +2^{-k n}\left\|f_{i}\right\|_{L^{p(\cdot)}\left(\mathbb{R}^{n}\right)}\left\|\chi_{i}\right\|_{L^{p^{(\cdot)}\left(\mathbb{R}^{n}\right)}} \\
\lesssim & 2^{-k n}\left\|f_{i}\right\|_{L^{p(\cdot)}\left(\mathbb{R}^{n}\right)}\left\|\chi_{B_{i}}\right\|_{L^{p^{\prime}(\cdot)}\left(\mathbb{R}^{n}\right)}
\end{aligned}
$$

Lemmas 7 and 8 lead to

$$
\begin{aligned}
& \left\|\mu_{\Omega}\left(f_{i}\right)(x) \chi_{k}\right\|_{L^{p(\cdot)}\left(\mathbb{R}^{n}\right)} \\
& \quad \leq 2^{-k n}\left\|f_{i}\right\|_{L^{p(\cdot)}\left(\mathbb{R}^{n}\right)}\left\|\chi_{B_{i}}\right\|_{L^{p^{\prime}(\cdot)}\left(\mathbb{R}^{n}\right)}\left\|\chi_{B_{k}}\right\|_{L^{p(\cdot)}\left(\mathbb{R}^{n}\right)} \\
& \quad \leq\left\|f_{i}\right\|_{L^{p(\cdot)}\left(\mathbb{R}^{n}\right)} \frac{\left\|\chi_{B_{i}}\right\|_{L^{p^{\prime}(\cdot)}\left(\mathbb{R}^{n}\right)}}{\left\|\chi_{B_{k}}\right\|_{L^{p^{\prime}(\cdot)}\left(\mathbb{R}^{n}\right)}} \\
& \quad \leq 2^{(i-k) n \delta_{2}}\left\|f_{i}\right\|_{L^{p(\cdot)}\left(\mathbb{R}^{n}\right)^{n}} .
\end{aligned}
$$

Thus we get

$$
\begin{aligned}
E_{<} & \leqslant\left\{\sum_{k=-\infty}^{-1} 2^{k \alpha(0) q}\left(\sum_{i=-\infty}^{k-2} 2^{(i-k) n \delta_{2}}\left\|f_{i}\right\|_{L^{p(\cdot)}\left(\mathbb{R}^{n}\right)}\right)^{q}\right\}^{1 / q} \\
& \approx\left\{\sum_{k=-\infty}^{-1}\left(\sum_{i=-\infty}^{k-2} 2^{\alpha(0) i}\left\|f_{i}\right\|_{L^{p(\cdot)}\left(\mathbb{R}^{n}\right)} 2^{(i-k)\left(n \delta_{2}-\alpha(0)\right)}\right)^{q}\right\}^{1 / q} .
\end{aligned}
$$

If $1<q<\infty$, since $n \delta_{2}-\alpha(0)>0$, Hölder's inequality implies that

$$
\begin{aligned}
E_{<} \lesssim & \left\{\sum_{k=-\infty}^{-1}\left(\sum_{i=-\infty}^{k-2} 2^{\alpha(0) i q}\left\|f_{i}\right\|_{L^{p(\cdot)}\left(\mathbb{R}^{n}\right)}^{q} 2^{(i-k)\left(n \delta_{2}-\alpha(0)\right) q / 2}\right)\right. \\
& \left.\times\left(\sum_{i=-\infty}^{k-2} 2^{(i-k)\left(n \delta_{2}-\alpha(0)\right) q^{\prime} / 2}\right)^{q / q^{\prime}}\right\}^{1 / q} \\
\lesssim & \left\{\sum_{i=-\infty}^{-3} 2^{\alpha(0) i q}\left\|f_{i}\right\|_{L^{p(\cdot)}\left(\mathbb{R}^{n}\right)}^{q} \sum_{k=i+2}^{-1} 2^{(i-k)\left(n \delta_{2}-\alpha(0)\right) q / 2}\right\}^{1 / q} \\
\lesssim & \left\|\left\{2^{\alpha(0) k} f \chi_{k}\right\}\right\|_{\ell_{<}^{q}\left(L^{p(\cdot)}\right)} \\
\lesssim & \|f\|_{\dot{K}_{p(\cdot)}^{\alpha(\cdot), q}\left(\mathbb{R}^{n}\right)} .
\end{aligned}
$$


If $0<q \leq 1$, by the well-known inequality

$$
\left(\sum_{i=1}^{\infty} a_{i}\right)^{q} \leq \sum_{i=1}^{\infty} a_{i}^{q} \quad\left(a_{i}>0, i=1,2, \ldots\right),
$$

we obtain

$$
\begin{aligned}
E_{<} & \lesssim\left\{\sum_{k=-\infty}^{-1} \sum_{i=-\infty}^{k-2} 2^{\alpha(0) i q}\left\|f_{i}\right\|_{L^{p(\cdot)}\left(\mathbb{R}^{n}\right)}^{q} 2^{(i-k)\left(n \delta_{2}-\alpha(0)\right) q}\right\}^{1 / q} \\
& \approx\left\{\sum_{i=-\infty}^{-3} 2^{\alpha(0) i q}\left\|f_{i}\right\|_{L^{p \cdot()}\left(\mathbb{R}^{n}\right)}^{q} \sum_{k=i+2}^{-1} 2^{(i-k)\left(n \delta_{2}-\alpha(0)\right) q}\right\}^{1 / q} \\
& \leq\left\|\left\{2^{\alpha(0) k} f \chi_{k}\right\}\right\|_{\ell_{<}^{q}\left(L^{p \cdot(\cdot)}\right)} \\
& \leqslant\|f\|_{\dot{K}_{p(\cdot)}^{\alpha(\cdot) q}}\left(\mathbb{R}^{n}\right)^{.}
\end{aligned}
$$

Similarly we have

$$
\begin{aligned}
E_{>} & \leqslant\left\{\sum_{k=0}^{\infty} 2^{k \alpha_{\infty} q}\left(\sum_{i=-\infty}^{k-2} 2^{(i-k) n \delta_{2}}\left\|f_{i}\right\|_{L^{p()}\left(\mathbb{R}^{n}\right)}\right)^{q}\right\}^{1 / q} \\
& \approx\left\{\sum_{k=0}^{\infty}\left(\sum_{i=-\infty}^{k-2} 2^{\alpha_{\infty} i}\left\|f_{i}\right\|_{L^{p())}\left(\mathbb{R}^{n}\right)} 2^{(i-k)\left(n \delta_{2}-\alpha_{\infty}\right)}\right)^{q}\right\}^{1 / q} .
\end{aligned}
$$

If $1<q<\infty$, since $\alpha_{\infty}+n \delta_{2}>2 \alpha_{\infty}>2 \alpha(0)$, then we get

$$
\begin{aligned}
& E_{>} \leq\left\{\sum_{k=0}^{\infty}\left(\sum_{i=-\infty}^{k-2} 2^{\alpha_{\infty} i q}\left\|f_{i}\right\|_{L^{p(\cdot)}\left(\mathbb{R}^{n}\right)}^{q} 2^{(i-k)\left(n \delta_{2}-\alpha_{\infty}\right) q / 2}\right)\right. \\
& \left.\times\left(\sum_{i=-\infty}^{k-2} 2^{(i-k)\left(n \delta_{2}-\alpha_{\infty}\right) q^{\prime} / 2}\right)^{q / q^{\prime}}\right\}^{1 / q} \\
& \lesssim\left\{\sum_{k=0}^{\infty}\left(\sum_{i=-\infty}^{k-2} 2^{\alpha_{\infty} i q}\left\|f_{i}\right\|_{L^{p(\cdot)}\left(\mathbb{R}^{n}\right)}^{q} 2^{(i-k)\left(n \delta_{2}-\alpha_{\infty}\right) q / 2}\right)\right\}^{1 / q} \\
& \approx\left\{\sum_{i=-\infty}^{-2} 2^{\alpha_{\infty} i q}\left\|f_{i}\right\|_{L^{p(\cdot)}\left(\mathbb{R}^{n}\right)}^{q} \sum_{k=0}^{\infty} 2^{(i-k)\left(n \delta_{2}-\alpha_{\infty}\right) q / 2}\right. \\
& \left.+\sum_{i=-1}^{\infty} 2^{\alpha_{\infty} i q}\left\|f_{i}\right\|_{L^{p(\cdot)}\left(\mathbb{R}^{n}\right)}^{q} \sum_{k=i+2}^{\infty} 2^{(i-k)\left(n \delta_{2}-\alpha_{\infty}\right) q / 2}\right\}^{1 / q} \\
& \lesssim\left\{\sum_{i=-\infty}^{-2} 2^{\alpha(0) i q} 2^{\left(\alpha_{\infty}+n \delta_{2}-2 \alpha(0)\right) i q / 2}\left\|f_{i}\right\|_{L^{p(\cdot)}\left(\mathbb{R}^{n}\right)}^{q}\right. \\
& \left.+\sum_{i=-1}^{\infty} 2^{\alpha_{\infty} i q}\left\|f_{i}\right\|_{L^{p(\cdot)}\left(\mathbb{R}^{n}\right)}^{q}\right\}^{1 / q} \\
& \lesssim\left\{\sum_{i=-\infty}^{-2} 2^{\alpha(0) i q}\left\|f_{i}\right\|_{L^{p(\cdot)}\left(\mathbb{R}^{n}\right)}^{q}+\sum_{i=-1}^{\infty} 2^{\alpha_{\infty} i q}\left\|f_{i}\right\|_{L^{p(\cdot)}\left(\mathbb{R}^{n}\right)}^{q}\right\}^{1 / q}
\end{aligned}
$$

$$
\begin{aligned}
& \leq\left\|\left\{2^{\alpha(0) k} f \chi_{k}\right\}\right\|_{\ell_{<}^{q}\left(L^{p(\cdot)}\right)}+\left\|\left\{2^{\alpha_{\infty} k} f \chi_{k}\right\}\right\|_{\ell_{>}^{q}\left(L^{p \cdot()}\right)} \\
& \approx\|f\|_{\dot{K}_{p(\cdot)}^{\alpha(\cdot),}\left(\mathbb{R}^{n}\right)} .
\end{aligned}
$$

If $0<q \leq 1$, since $\alpha(0) \leq \alpha_{\infty}$, we obtain

$$
\begin{aligned}
& E_{>} \lesssim\left\{\sum_{k=0}^{\infty} \sum_{i=-\infty}^{k-2} 2^{\alpha_{\infty} i q}\left\|f_{i}\right\|_{L^{p \cdot()}\left(\mathbb{R}^{n}\right)}^{q} 2^{(i-k)\left(n \delta_{2}-\alpha_{\infty}\right) q}\right\}^{1 / q} \\
& \approx\left\{\sum_{i=-\infty}^{-2} 2^{\alpha_{\infty} i q}\left\|f_{i}\right\|_{L^{p(\cdot)}\left(\mathbb{R}^{n}\right)}^{q} \sum_{k=0}^{\infty} 2^{(i-k)\left(n \delta_{2}-\alpha_{\infty}\right) q}\right. \\
& \left.+\sum_{i=-1}^{\infty} 2^{\alpha_{\infty} i q}\left\|f_{i}\right\|_{L^{p \cdot()}\left(\mathbb{R}^{n}\right)}^{q} \sum_{k=i+2}^{\infty} 2^{(i-k)\left(n \delta_{2}-\alpha_{\infty}\right) q}\right\}^{1 / q} \\
& \lesssim\left\{\sum_{i=-\infty}^{-2} 2^{\alpha(0) i q}\left\|f_{i}\right\|_{L^{p(\cdot)}\left(\mathbb{R}^{n}\right)}^{q}+\sum_{i=-1}^{\infty} 2^{\alpha_{\infty} i q}\left\|f_{i}\right\|_{L^{p(\cdot)}\left(\mathbb{R}^{n}\right)}^{q}\right\}^{1 / q} \\
& \lesssim\left\|\left\{2^{\alpha(0) k} f \chi_{k}\right\}\right\|_{\ell_{-}^{q}\left(L^{p(\cdot)}\right)}+\left\|\left\{2^{\alpha_{\infty} k} f \chi_{k}\right\}\right\|_{\ell_{>}^{q}\left(L^{p(\cdot)}\right)} \\
& \approx\|f\|_{\dot{K}_{p(\cdot)}^{\alpha(\cdot), q}\left(\mathbb{R}^{n}\right)} .
\end{aligned}
$$

Thus, we arrive at

$$
E=E_{<}+E_{>} \lesssim\|f\|_{\dot{K}_{p(\cdot)}^{\alpha(\cdot), q}\left(\mathbb{R}^{n}\right)} .
$$

For $G$, observe that if $x \in R_{k}, y \in R_{i}$, and $i \geq k+2$, then $|x-y| \approx|y| \approx 2^{i}$ and

$$
\left|\frac{1}{|x-y|^{2}}-\frac{1}{|y|^{2}}\right| \leqslant \frac{|x|}{|x-y|^{3}} .
$$

From Minkowski's inequality and Lemma 6, it follows that

$$
\begin{aligned}
& \left|\mu_{\Omega}\left(f_{i}\right)(x)\right| \\
& \leq \quad\left(\int_{0}^{|y|}\left|\int_{|x-y| \leq t} \frac{\Omega(x-y)}{|x-y|^{n-1}} f_{i}(y) d y\right|^{2} \frac{d t}{t^{3}}\right)^{1 / 2} \\
& \quad+\left(\int_{|y|}^{\infty}\left|\int_{|x-y| \leq t} \frac{\Omega(x-y)}{|x-y|^{n-1}} f_{i}(y) d y\right|^{2} \frac{d t}{t^{3}}\right)^{1 / 2} \\
& \leq \int_{R_{i}} \frac{\left|f_{i}(y)\right|}{|x-y|^{n-1}}\left(\int_{|x-y| \leq t,|y| \geq t} \frac{d t}{t^{3}}\right)^{1 / 2} d y \\
& \quad+\int_{R_{i}} \frac{\left|f_{i}(y)\right|}{|x-y|^{n-1}}\left(\int_{|y|}^{\infty} \frac{d t}{t^{3}}\right)^{1 / 2} d y \\
& \leq \int_{R_{i}} \frac{\left|f_{i}(y)\right|}{|x-y|^{n-1}} \cdot \frac{|x|^{1 / 2}}{|x-y|^{3 / 2}} d y+\int_{R_{i}} \frac{\left|f_{i}(y)\right|}{|x-y|^{n-1}} \cdot \frac{1}{|y|} d y
\end{aligned}
$$


6

Journal of Function Spaces

$$
\begin{aligned}
\lesssim & 2^{(k-i) / 2} 2^{-i n}\left\|f_{i}\right\|_{L^{p(\cdot)}\left(\mathbb{R}^{n}\right)}\left\|\chi_{i}\right\|_{L^{p^{\prime}(\cdot)}\left(\mathbb{R}^{n}\right)} \\
& +C 2^{-i n}\left\|f_{i}\right\|_{L^{p(\cdot)}\left(\mathbb{R}^{n}\right)}\left\|\chi_{i}\right\|_{L^{p^{\prime}(\cdot)}\left(\mathbb{R}^{n}\right)} \\
\lesssim & 2^{-i n}\left\|f_{i}\right\|_{L^{p \cdot(\cdot)}\left(\mathbb{R}^{n}\right)}\left\|\chi_{B_{i}}\right\|_{L^{p^{\prime} \cdot(\cdot)}\left(\mathbb{R}^{n}\right)^{n}} .
\end{aligned}
$$

By Lemmas 7 and 8, we have

$$
\begin{aligned}
& \left\|\mu_{\Omega}\left(f_{i}\right)(x) \chi_{k}\right\|_{L^{p(\cdot)}\left(\mathbb{R}^{n}\right)} \\
& \quad \leqslant 2^{-i n}\left\|f_{i}\right\|_{L^{p(\cdot)}\left(\mathbb{R}^{n}\right)}\left\|\chi_{B_{i}}\right\|_{L^{p^{\prime}(\cdot)}\left(\mathbb{R}^{n}\right)}\left\|\chi_{B_{k}}\right\|_{L^{p(\cdot)}\left(\mathbb{R}^{n}\right)} \\
& \lesssim\left\|f_{i}\right\|_{L^{p(\cdot)}\left(\mathbb{R}^{n}\right)} \frac{\left\|\chi_{B_{k}}\right\|_{L^{p(\cdot)}\left(\mathbb{R}^{n}\right)}}{\left\|\chi_{B_{i}}\right\|_{L^{p(\cdot)}\left(\mathbb{R}^{n}\right)}} \\
& \quad \lesssim 2^{(k-i) n \delta_{1}}\left\|f_{i}\right\|_{L^{p \cdot()}\left(\mathbb{R}^{n}\right)^{\cdot}}
\end{aligned}
$$

Thus we get

$$
\begin{aligned}
G_{<} & \lesssim\left\{\sum_{k=-\infty}^{-1} 2^{k \alpha(0) q}\left(\sum_{i=k+2}^{\infty} 2^{(k-i) n \delta_{1}}\left\|f_{i}\right\|_{L^{p \cdot()}\left(\mathbb{R}^{n}\right)}\right)^{q}\right\}^{1 / q} \\
& \approx\left\{\sum_{k=-\infty}^{-1}\left(\sum_{i=k+2}^{\infty} 2^{\alpha(0) i}\left\|f_{i}\right\|_{L^{p(\cdot)}\left(\mathbb{R}^{n}\right)} 2^{(k-i)\left(n \delta_{1}+\alpha(0)\right)}\right)^{q}\right\}^{1 / q}, \\
G_{>} & \lesssim\left\{\sum_{k=0}^{\infty}\left(\sum_{i=k+2}^{\infty} 2^{\alpha_{\infty} i}\left\|f_{i}\right\|_{L^{p(\cdot)}\left(\mathbb{R}^{n}\right)} 2^{(k-i)\left(n \delta_{1}+\alpha_{\infty}\right)}\right)^{q}\right\}^{1 / q} .
\end{aligned}
$$

Using the same arguments as that for $E_{<}$and $E_{>}$, we get

$$
G=G_{<}+G_{>} \lesssim\|f\|_{\dot{K}_{p(\cdot)}^{\alpha(\cdot), q}\left(\mathbb{R}^{n}\right)}
$$

Hence the proof of Theorem 11 is completed.

Proof of Theorem 12. We apply Proposition 5 again and get

$$
\begin{aligned}
\left\|\mu_{\Omega, b}^{m}(f)\right\|_{\dot{K}_{p(\cdot)}^{\alpha(\cdot), q}\left(\mathbb{R}^{n}\right)} \approx & \left\|\left\{2^{\alpha(0) k} \mu_{\Omega, b}^{m}(f) \chi_{k}\right\}\right\|_{\ell_{<}^{q}\left(L^{p(\cdot)}\right)} \\
& +\left\|\left\{2^{\alpha_{\infty} k} \mu_{\Omega, b}^{m}(f) \chi_{k}\right\}\right\|_{\ell_{>}^{q}\left(L^{p(\cdot)}\right)} \\
= & J_{<}+J_{>} .
\end{aligned}
$$

Let $f \in \dot{K}_{p(\cdot)}^{\alpha(\cdot), q}\left(\mathbb{R}^{n}\right)$, and write

$$
f(x)=\sum_{i=-\infty}^{\infty} f(x) \chi_{i}(x)=\sum_{i=-\infty}^{\infty} f_{i}(x)
$$

By Minkowski’s inequality, we have

$$
\begin{aligned}
J_{<}= & \left\{\sum_{k=-\infty}^{-1} 2^{\alpha(0) k q}\left\|\mu_{\Omega, b}^{m}(f) \chi_{k}\right\|_{L^{p(\cdot)}\left(\mathbb{R}^{n}\right)}^{q}\right\}^{1 / q} \\
\leq & \left\{\sum_{k=-\infty}^{-1} 2^{\alpha(0) k q}\left(\sum_{i=-\infty}^{k-2}\left\|\mu_{\Omega, b}^{m}\left(f_{i}\right) \chi_{k}\right\|_{L^{p(\cdot)}\left(\mathbb{R}^{n}\right)}\right)^{q}\right\}^{1 / q} \\
& +\left\{\sum_{k=-\infty}^{-1} 2^{\alpha(0) k q}\left(\sum_{i=k-1}^{k+1}\left\|\mu_{\Omega, b}^{m}\left(f_{i}\right) \chi_{k}\right\|_{L^{p(\cdot)}\left(\mathbb{R}^{n}\right)}\right)^{q}\right\}^{1 / q} \\
& +\left\{\sum_{k=-\infty}^{-1} 2^{\alpha(0) k q}\left(\sum_{i=k+2}^{\infty}\left\|\mu_{\Omega, b}^{m}\left(f_{i}\right) \chi_{k}\right\|_{L^{p(\cdot)}\left(\mathbb{R}^{n}\right)}\right)^{q}\right\}^{1 / q} \\
= & U_{<}+V_{<}+W_{<} .
\end{aligned}
$$

By the same way, we obtain

$$
\begin{aligned}
J_{>}= & \left\{\sum_{k=0}^{\infty} 2^{\alpha_{\infty} k q}\left\|\mu_{\Omega, b}^{m}(f) \chi_{k}\right\|_{L^{p(\cdot)}\left(\mathbb{R}^{n}\right)}^{q}\right\}^{1 / q} \\
\lesssim & \left\{\sum_{k=0}^{\infty} 2^{\alpha_{\infty} k q}\left(\sum_{i=-\infty}^{k-2}\left\|\mu_{\Omega, b}^{m}\left(f_{i}\right) \chi_{k}\right\|_{L^{p(\cdot)}\left(\mathbb{R}^{n}\right)}\right)^{q}\right\}^{1 / q} \\
& +\left\{\sum_{k=0}^{\infty} 2^{\alpha_{\infty} k q}\left(\sum_{i=k-1}^{k+1}\left\|\mu_{\Omega, b}^{m}\left(f_{i}\right) \chi_{k}\right\|_{L^{p(\cdot)}\left(\mathbb{R}^{n}\right)}\right)^{q}\right\}^{1 / q} \\
& +\left\{\sum_{k=0}^{\infty} 2^{\alpha_{\infty} k q}\left(\sum_{i=k+2}^{\infty}\left\|\mu_{\Omega, b}^{m}\left(f_{i}\right) \chi_{k}\right\|_{L^{p(\cdot)}\left(\mathbb{R}^{n}\right)}\right)^{q}\right\}^{1 / q} \\
= & U_{>}+V_{>}+W_{>} .
\end{aligned}
$$

Thus, we have

$$
\left\|\mu_{\Omega, b}^{m}(f)\right\|_{\dot{K}_{p(\cdot)}^{\alpha(\cdot), q}\left(\mathbb{R}^{n}\right)} \leqslant U+V+W
$$

where $U=U_{<}+U_{>}, V=V_{<}+V_{>}$, and $W=W_{<}+W_{>}$. For $V$, by Lemma 10, we have

$$
\begin{aligned}
V= & V_{<}+V_{>} \\
\lesssim & \left\{\sum_{k=-\infty}^{-1} 2^{\alpha(0) k q}\left\|f_{k}\right\|_{L^{p(\cdot)}\left(\mathbb{R}^{n}\right)}^{q}\right\}^{1 / q} \\
& +\left\{\sum_{k=0}^{\infty} 2^{\alpha_{\infty} k q}\left\|f_{k}\right\|_{L^{p(\cdot)}\left(\mathbb{R}^{n}\right)}^{q}\right\}^{1 / q} \\
\lesssim & \left\|\left\{2^{\alpha(0) k} f \chi_{k}\right\}\right\|_{\ell_{<}^{q}\left(L^{p(\cdot)}\right)}+\left\|\left\{2^{\alpha_{\infty} k} f \chi_{k}\right\}\right\|_{\ell_{>}^{q}\left(L^{p(\cdot)}\right)} \\
\approx & \|f\|_{\dot{K}_{p(\cdot)}^{\alpha(\cdot), q}\left(\mathbb{R}^{n}\right)} .
\end{aligned}
$$


Journal of Function Spaces

7

For $U$, observe that if $x \in R_{k}, y \in R_{i}$, and $i \leq k-2$, then

$$
\begin{aligned}
& \left|\mu_{\Omega, b}^{m}\left(f_{i}\right)(x)\right| \\
& \lesssim\left(\int_{0}^{|x|}\left|\int_{|x-y| \leq t} \frac{\Omega(x-y)}{|x-y|^{n-1}}[b(x)-b(y)]^{m} f_{i}(y) d y\right|^{2} \frac{d t}{t^{3}}\right)^{1 / 2} \\
& +\left(\int_{|x|}^{\infty}\left|\int_{|x-y| \leq t} \frac{\Omega(x-y)}{|x-y|^{n-1}}[b(x)-b(y)]^{m} f_{i}(y) d y\right|^{2} \frac{d t}{t^{3}}\right)^{1 / 2} \\
& \lesssim \int_{R_{i}} \frac{|b(x)-b(y)|^{m}\left|f_{i}(y)\right|}{|x-y|^{n-1}}\left(\int_{|x-y| \leq t,|x| \geq t} \frac{d t}{t^{3}}\right)^{1 / 2} d y \\
& +\int_{R_{i}} \frac{|b(x)-b(y)|^{m}\left|f_{i}(y)\right|}{|x-y|^{n-1}}\left(\int_{|x|}^{\infty} \frac{d t}{t^{3}}\right)^{1 / 2} d y \\
& \lesssim \int_{R_{i}} \frac{|b(x)-b(y)|^{m}\left|f_{i}(y)\right|}{|x-y|^{n-1}} \cdot \frac{|y|^{1 / 2}}{|x-y|^{3 / 2}} d y \\
& +\int_{R_{i}} \frac{|b(x)-b(y)|^{m}\left|f_{i}(y)\right|}{|x-y|^{n-1}} \cdot \frac{1}{|x|} d y \\
& \lesssim 2^{(i-k) / 2} 2^{-k n} \int_{R_{i}}|b(x)-b(y)|^{m}\left|f_{i}(y)\right| d y \\
& +2^{-k n} \int_{R_{i}}|b(x)-b(y)|^{m}\left|f_{i}(y)\right| d y \\
& \lesssim 2^{-k n} \int_{R_{i}}|b(x)-b(y)|^{m}\left|f_{i}(y)\right| d y \\
& \lesssim 2^{-k n} \sum_{j=0}^{m} C_{m}^{j}\left|b(x)-b_{B_{i}}\right|^{m-j} \int_{R_{i}}\left|b_{B_{i}}-b(y)\right|^{j}\left|f_{i}(y)\right| d y \\
& \lesssim 2^{-k n}\left\|f_{i}\right\|_{L^{p(\cdot)}\left(\mathbb{R}^{n}\right)} \sum_{j=0}^{m} C_{m}^{j}\left|b(x)-b_{B_{i}}\right|^{m-j}\left\|\left(b_{B_{i}}-b\right)^{j} \chi_{i}\right\|_{L^{p^{\prime}(\cdot)}\left(\mathbb{R}^{n}\right)} .
\end{aligned}
$$

An application of Lemmas 7, 8, and 10 gives

$$
\begin{aligned}
& \left\|\mu_{\Omega, b}^{m}\left(f_{i}\right) \chi_{k}\right\|_{L^{p(\cdot)}\left(\mathbb{R}^{n}\right)} \\
& \leqslant 2^{-k n}\left\|f_{i}\right\|_{L^{p(\cdot)}\left(\mathbb{R}^{n}\right)} \\
& \quad \times \sum_{j=0}^{m} C_{m}^{j}\left\|\left(b(x)-b_{B_{i}}\right)^{m-j} \chi_{k}\right\|_{L^{p(\cdot)}\left(\mathbb{R}^{n}\right)}\left\|\left(b_{B_{i}}-b\right)^{j} \chi_{i}\right\|_{L^{p^{\prime}(\cdot)}\left(\mathbb{R}^{n}\right)} \\
& \leqslant 2^{-k n}\left\|f_{i}\right\|_{L^{p(\cdot)}\left(R^{n}\right)} \\
& \quad \times \sum_{j=0}^{m} C_{m}^{j}(k-i)^{m-j}\|b\|_{\mathrm{BMO}}^{m-j}\left\|\chi_{B_{k}}\right\|_{L^{p(\cdot)}\left(\mathbb{R}^{n}\right)}\|b\|_{\mathrm{BMO}}^{j}\left\|\chi_{B_{i}}\right\|_{L^{p^{\prime}(\cdot)}\left(\mathbb{R}^{n}\right)} \\
& \leqslant(k-i+1)^{m} 2^{-k n}\left\|f_{i}\right\|_{L^{p(\cdot)}\left(\mathbb{R}^{n}\right)}\left\|\chi_{B_{k}}\right\|\left\|_{L^{p(\cdot)}\left(\mathbb{R}^{n}\right)}\right\| \chi_{B_{i}}\|\|_{L^{p^{\prime}(\cdot)}\left(\mathbb{R}^{n}\right)}
\end{aligned}
$$

$$
\begin{aligned}
& \lesssim(k-i+1)^{m}\left\|f_{i}\right\|_{L^{p \cdot(\cdot)}\left(\mathbb{R}^{n}\right)} \frac{\left\|\chi_{B_{i}}\right\|_{L^{p^{\prime} \cdot()}\left(\mathbb{R}^{n}\right)}}{\left\|\chi_{B_{k}}\right\|_{L^{p^{(\cdot)}\left(\mathbb{R}^{n}\right)}}} \\
& \leqslant(k-i+1)^{m} 2^{(i-k) n \delta_{2}}\left\|f_{i}\right\|_{L^{p(\cdot)}\left(\mathbb{R}^{n}\right)^{\cdot}}
\end{aligned}
$$

For convenience below we put $\sigma=n \delta_{2}-\alpha(0)$; if $1<q<$ $\infty$, then we use Hölder's inequality and obtain

$$
\begin{aligned}
U_{<} & \lesssim\left\{\sum_{k=-\infty}^{-1}\left(\sum_{i=-\infty}^{k-2} 2^{\alpha(0) i}\left\|f_{i}\right\|_{L^{p(\cdot)}\left(\mathbb{R}^{n}\right)}(k-i+1)^{m} 2^{(i-k) \sigma}\right)^{q}\right\}^{1 / q} \\
& \lesssim\left\{\sum_{k=-\infty}^{-1}\left(\sum_{i=-\infty}^{k-2} 2^{\alpha(0) i q}\left\|f_{i}\right\|_{L^{p(\cdot)}\left(\mathbb{R}^{n}\right)}^{q} 2^{(i-k) \sigma q / 2}\right)\right. \\
& \left.\times\left(\sum_{i=-\infty}^{k-2}(k-i+1)^{m q^{\prime}} 2^{(i-k) \sigma q^{\prime} / 2}\right)^{q / q^{\prime}}\right\}^{1 / q} \\
& \left\{\left\{\sum_{i=-\infty}^{-3} 2^{\alpha(0) i q}\left\|f_{i}\right\|_{L^{p(\cdot)}\left(\mathbb{R}^{n}\right)}^{q} \sum_{k=i+2}^{-1} 2^{(i-k) \sigma q / 2}\right\}^{1 / q}\right. \\
& \leqslant\left\|\left\{2^{\alpha(0) k} f \chi_{k}\right\}\right\|_{\ell_{<}^{q}\left(L^{p(\cdot)}\right)} \\
& \leqslant f \|_{\dot{K}_{p(\cdot)}^{\alpha(\cdot), q}\left(\mathbb{R}^{n}\right)^{.}}
\end{aligned}
$$

If $0<q \leq 1$, then we get

$$
\begin{aligned}
U_{<} & \lesssim\left\{\sum_{k=-\infty}^{-1} \sum_{i=-\infty}^{k-2} 2^{\alpha(0) i q}\left\|f_{i}\right\|_{L^{p(\cdot)}\left(\mathbb{R}^{n}\right)}^{q}(k-i+1)^{m q} 2^{(i-k) \sigma q}\right\}^{1 / q} \\
& \approx\left\{\sum_{i=-\infty}^{-3} 2^{\alpha(0) i q}\left\|f_{i}\right\|_{L^{p(\cdot)}\left(\mathbb{R}^{n}\right)}^{q} \sum_{k=i+2}^{-1}(k-i+1)^{m q} 2^{(i-k) \sigma q}\right\}^{1 / q} \\
& \lesssim\left\|\left\{2^{\alpha(0) k} f \chi_{k}\right\}\right\|_{\ell_{<}^{q}\left(L^{p(\cdot)}\right)} \\
& \lesssim\|f\|_{\dot{K}_{p(\cdot)}^{\alpha(\cdot),}\left(\mathbb{R}^{n}\right)} .
\end{aligned}
$$

Similarly, we put $\eta=n \delta_{2}-\alpha_{\infty}$; if $1<q<\infty$, by Hölder's inequality, we obtain

$$
\begin{aligned}
U_{>} \lesssim & \left\{\sum_{k=0}^{\infty}\left(\sum_{i=-\infty}^{k-2} 2^{\alpha_{\infty} i}\left\|f_{i}\right\|_{L^{p(\cdot)}\left(\mathbb{R}^{n}\right)}(k-i+1)^{m} 2^{(i-k) \eta}\right)^{q}\right\}^{1 / q} \\
\lesssim & \left\{\sum_{k=0}^{\infty}\left(\sum_{i=-\infty}^{k-2} 2^{\alpha_{\infty} i q}\left\|f_{i}\right\|_{L^{p(\cdot)}\left(\mathbb{R}^{n}\right)}^{q} 2^{(i-k) \eta q / 2}\right)\right. \\
& \left.\times\left(\sum_{i=-\infty}^{k-2}(k-i+1)^{m q^{\prime}} 2^{(i-k) \eta q^{\prime} / 2}\right)^{q / q^{\prime}}\right\}^{1 / q} \\
\lesssim & \left\{\sum_{k=0}^{\infty}\left(\sum_{i=-\infty}^{k-2} 2^{\alpha_{\infty} i q}\left\|f_{i}\right\|_{L^{p(\cdot)}\left(\mathbb{R}^{n}\right)}^{q} 2^{(i-k) \eta q / 2}\right)\right\}^{1 / q} .
\end{aligned}
$$


By the same arguments as $E_{>}$, we get

$$
\begin{aligned}
U_{>} & \lesssim\left\|\left\{2^{\alpha(0) k} f \chi_{k}\right\}\right\|_{\ell_{<}^{q}\left(L^{p(\cdot)}\right)}+\left\|\left\{2^{\alpha_{\infty} k} f \chi_{k}\right\}\right\|_{\ell_{>}^{q}\left(L^{p(\cdot)}\right)} \\
& \approx\|f\|_{\dot{K}_{p(\cdot)}^{\alpha(\cdot), q}\left(\mathbb{R}^{n}\right)^{n}} .
\end{aligned}
$$

If $0<q \leq 1$, we obtain

$$
\begin{aligned}
U_{>} & \leqslant\left\{\sum_{k=0}^{\infty} \sum_{i=-\infty}^{k-2} 2^{\alpha_{\infty} i q}\left\|f_{i}\right\|_{L^{p(\cdot)}\left(\mathbb{R}^{n}\right)}^{q}(k-i+1)^{m q} 2^{(i-k) \eta q}\right\}^{1 / q} \\
\approx & \left\{\sum_{i=-\infty}^{-2} 2^{\alpha_{\infty} i q}\left\|f_{i}\right\|_{L^{p(\cdot)}\left(\mathbb{R}^{n}\right)}^{q} \sum_{k=0}^{\infty}(k-i+1)^{m q} 2^{(i-k) \eta q}\right. \\
& \left.+\sum_{i=-1}^{\infty} 2^{\alpha \alpha_{\infty} i q}\left\|f_{i}\right\|_{L^{p(\cdot)}\left(\mathbb{R}^{n}\right)}^{q} \sum_{k=i+2}^{\infty}(k-i+1)^{m q} 2^{(i-k) \eta q}\right\}^{1 / q} \\
\lesssim & \left\{\sum_{i=-\infty}^{-2} 2^{\alpha(0) i q}\left\|f_{i}\right\|_{L^{p(\cdot)}\left(\mathbb{R}^{n}\right)}^{q}+\sum_{i=-1}^{\infty} 2^{\alpha \alpha_{\infty} i q}\left\|f_{i}\right\|_{L^{p(\cdot)}\left(\mathbb{R}^{n}\right)}^{q}\right\}^{1 / q} \\
\lesssim & \left\|\left\{2^{\alpha(0) k} f \chi_{k}\right\}\right\|_{\ell_{<}^{q}\left(L^{p(\cdot)}\right)}+\left\|\left\{2^{\alpha_{\infty} k} f \chi_{k}\right\}\right\|_{\ell_{>}^{q}\left(L^{p(\cdot)}\right)} \\
\approx & \|f\|_{\dot{K}_{p(\cdot)}^{\alpha(\cdot), q}\left(\mathbb{R}^{n}\right)}
\end{aligned}
$$

Thus, we have

$$
U=U_{<}+U_{>} \lesssim\|f\|_{\dot{K}_{p(\cdot)}^{\alpha(\cdot), q}\left(\mathbb{R}^{n}\right)^{\cdot}}
$$

For $W$, if $x \in R_{k}, y \in R_{i}$, and $i \geq k+2$, as in the arguments for $G$ and $U$, we obtain

$$
\left\|\mu_{\Omega, b}^{m}\left(f_{j}\right) \chi_{k}\right\|_{L^{p(\cdot)}\left(\mathbb{R}^{n}\right)} \lesssim(i-k+1)^{m} 2^{(k-i) n \delta_{1}}\left\|f_{j}\right\|_{L^{p(\cdot)}\left(\mathbb{R}^{n}\right)^{\circ}} .
$$

Thus we get

$W_{<}$

$$
\begin{gathered}
\leq\left\{\sum_{k=-\infty}^{-1} 2^{k \alpha(0) q}\left(\sum_{i=k+2}^{\infty}(i-k+1)^{m} 2^{(k-i) n \delta_{1}}\left\|f_{i}\right\|_{L^{p(\cdot)}\left(\mathbb{R}^{n}\right)}\right)^{q}\right\}^{1 / q} \\
\approx\left\{\sum _ { k = - \infty } ^ { - 1 } \left(\sum_{i=k+2}^{\infty} 2^{\alpha(0) i}\left\|f_{i}\right\|_{L^{p(\cdot)}\left(\mathbb{R}^{n}\right)}\right.\right. \\
\left.\left.\times(i-k+1)^{m} 2^{(k-i)\left(n \delta_{1}+\alpha(0)\right)}\right)^{q}\right\}^{1 / q}
\end{gathered}
$$

$W_{>}$

$$
\lesssim\left\{\sum_{k=0}^{\infty}\left(\sum_{i=k+2}^{\infty} 2^{\alpha_{\infty} i}\left\|f_{i}\right\|_{L^{p(\cdot)}\left(\mathbb{R}^{n}\right)}(i-k+1)^{m} 2^{(k-i)\left(n \delta_{1}+\alpha_{\infty}\right)}\right)^{q}\right\}^{1 / q} .
$$

Similar to the estimates of $U_{<}$and $U_{>}$, we get

$$
\left.W=W_{<}+W_{>} \lesssim\|f\|_{\dot{K}_{p(\cdot)}^{\alpha(\cdot), q}} \mathbb{R}^{n}\right)^{.}
$$

Hence the proof of Theorem 12 is completed.

\section{Conflict of Interests}

The author declares that there is no conflict of interests regarding the publication of this paper.

\section{Acknowledgments}

The author would like to thank the referees for their time and valuable comments. This work was supported by the NSF of China (Grant no. 11201003) and University NSR Project of Anhui Province (Grant no. KJ2014A087).

\section{References}

[1] E. M. Stein, "On the functions of Littlewood-Paley, Lusin, and Marcinkiewicz," Transactions of the American Mathematical Society, vol. 88, pp. 430-466, 1958.

[2] A. Benedek, A.-P. Calderón, and R. Panzone, "Convolution operators on Banach space valued functions," Proceedings of the National Academy of Sciences of the United States of America, vol. 48, pp. 356-365, 1962.

[3] Y. Ding, D. Fan, and Y. Pan, "Weighted boundedness for a class of rough Marcinkiewicz integrals," Indiana University Mathematics Journal, vol. 48, no. 3, pp. 1037-1055, 1999.

[4] A. Torchinsky and S. L. Wang, "A note on the Marcinkiewicz integral," Colloquium Mathematicum, vol. 60-61, no. 1, pp. 235243, 1990.

[5] Y. Ding, S. Lu, and K. Yabuta, "On commutators of Marcinkiewicz integrals with rough kernel," Journal of Mathematical Analysis and Applications, vol. 275, no. 1, pp. 60-68, 2002.

[6] Y. Ding, S. Lu, and P. Zhang, "Weighted weak type estimates for commutators of the Marcinkiewicz integrals," Science in China A, vol. 47, no. 1, pp. 83-95, 2004.

[7] P. Zhang, "Weighted endpoint estimates for commutators of Marcinkiewicz integrals," Acta Mathematica Sinica, vol. 26, no. 9, pp. 1709-1722, 2010.

[8] S. Lu, "Marcinkiewicz integral with rough kernels," Frontiers of Mathematics in China, vol. 3, no. 1, pp. 1-14, 2008.

[9] Y. P. Chen and Y. Ding, " $L^{p}$ boundedness of the commutators of Marcinkiewicz integrals with rough kernels," Forum Mathematicum, 2013.

[10] Y. M. Chen, S. Levine, and M. Rao, "Variable exponent, linear growth functionals in image restoration," SIAM Journal on Applied Mathematics, vol. 66, no. 4, pp. 1383-1406, 2006.

[11] P. Harjulehto, P. Hästö, Ú. V. Lê, and M. Nuortio, "Overview of differential equations with non-standard growth," Nonlinear Analysis: Theory, Methods \& Applications, vol. 72, no. 12, pp. 4551-4574, 2010

[12] O. Kováčik and J. Rákosník, "On spaces $L^{p(x)}$ and $W^{k, p(x), "}$ Czechoslovak Mathematical Journal, vol. 41, no. 4, pp. 592-618, 1991.

[13] D. Cruz-Uribe, A. Fiorenza, J. M. Martell, and C. Pérez, "The boundedness of classical operators on variable $L^{p}$ spaces," Annales Academiae Scientiarum Fennicae Mathematica, vol. 31, no. 1, pp. 239-264, 2006.

[14] L. Diening, P. Harjulehto, P. Hästö, and M. Ruzicka, Lebesgue and Sobolev Spaces with Variable Exponents, vol. 2017 of Lecture Notes in Mathematics, Springer, Heidelberg, Germany, 2011.

[15] D. V. Cruz-Uribe and A. Fiorenza, Variable Lebesgue Spaces: Foundations and Harmonic Analysis, Applied and Numerical Harmonic Analysis, Birkhäuser, Basel, Switzerland, 2013. 
[16] M. Izuki, "Herz and amalgam spaces with variable exponent, the Haar wavelets and greediness of the wavelet system," East Journal on Approximations, vol. 15, no. 1, pp. 87-109, 2009.

[17] M. Izuki, "Commutators of fractional integrals on Lebesgue and Herz spaces with variable exponent," Rendiconti del Circolo Matematico di Palermo: Second Series, vol. 59, no. 3, pp. 461-472, 2010.

[18] H. B. Wang, Z. W. Fu, and Z. G. Liu, "Higher-order commutators of Marcinkiewicz integrals on variable Lebesgue spaces," Acta Mathematica Scientia A, vol. 32, no. 6, pp. 1092-1101, 2012.

[19] Z. G. Liu and H. B. Wang, "Boundedness of Marcinkiewicz integrals on Herz spaces with variable exponent," The Jordanian Journal of Mathematics and Statistics, vol. 5, no. 4, pp. 223-239, 2012.

[20] A. Almeida and D. Drihem, "Maximal, potential and singular type operators on Herz spaces with variable exponents," Journal of Mathematical Analysis and Applications, vol. 394, no. 2, pp. 781-795, 2012.

[21] S. Z. Lu, D. C. Yang, and G. E. Hu, Herz Type Spaces and Their Applications, Science Press, Beijing, China, 2008.

[22] X. W. Li and D. C. Yang, "Boundedness of some sublinear operators on Herz spaces," Illinois Journal of Mathematics, vol. 40, no. 3, pp. 484-501, 1996.

[23] M. Izuki, "Vector-valued inequalities on HERz spaces and characterizations of HERz-Sobolev spaces with variable exponent," Glasnik Matematički, vol. 45, no. 65, pp. 475-503, 2010.

[24] L. Wang and L. Shu, "Higher order commutators of Marcinkiewicz integral operator on Herz-Morrey spaces with variable exponent," Journal of Mathematical Research with Applications, vol. 34, no. 2, pp. 175-186, 2014.

[25] B. Dong and J. Xu, "New Herz type Besov and Triebel-Lizorkin spaces with variable exponents," Journal of Function Spaces and Applications, vol. 2012, Article ID 384593, 27 pages, 2012. 


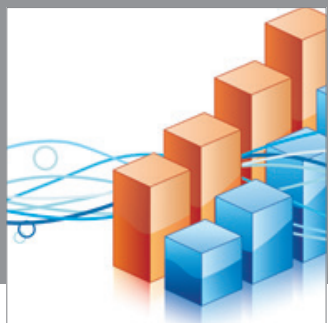

Advances in

Operations Research

mansans

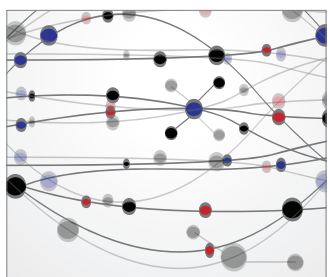

The Scientific World Journal
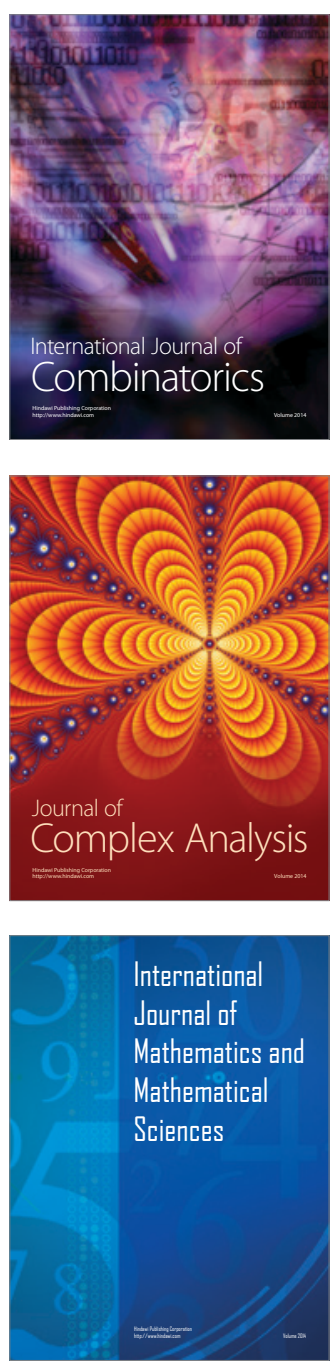
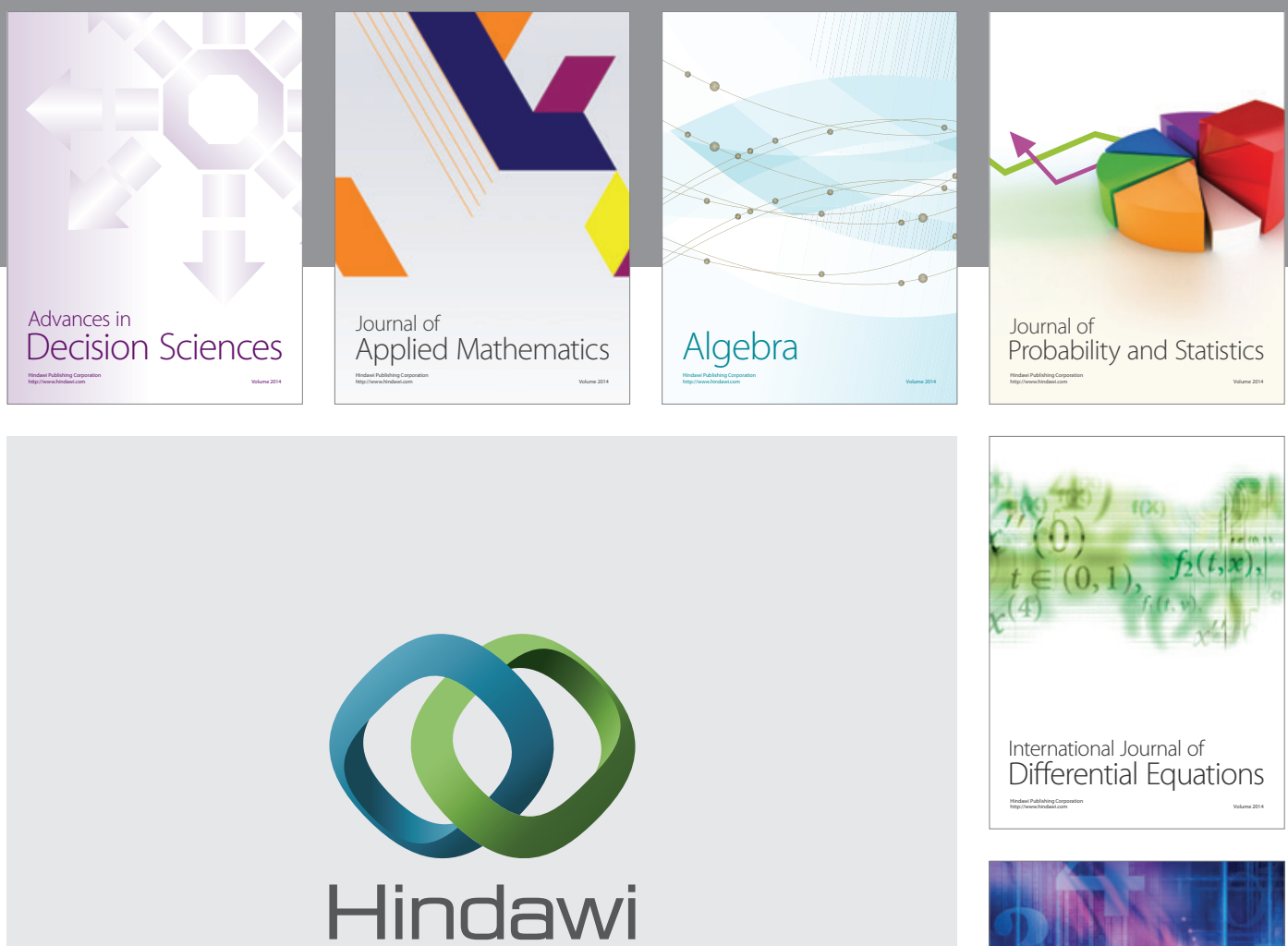

Submit your manuscripts at http://www.hindawi.com
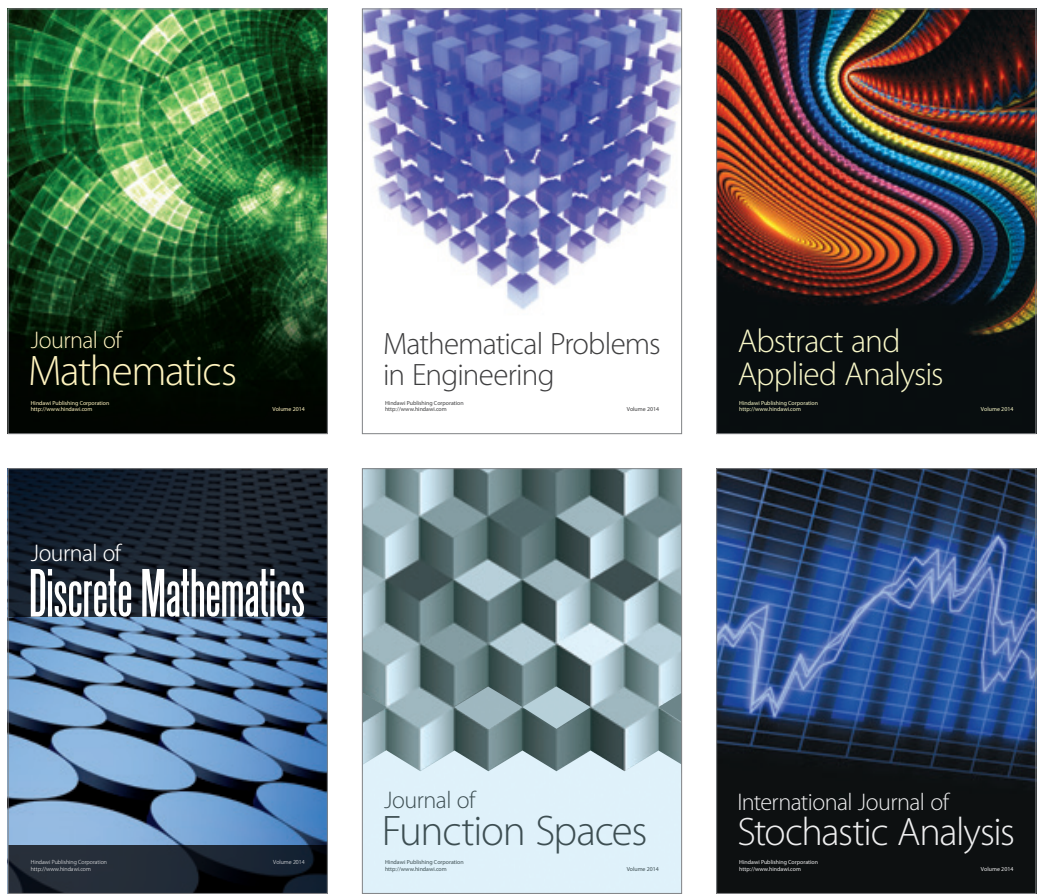

Journal of

Function Spaces

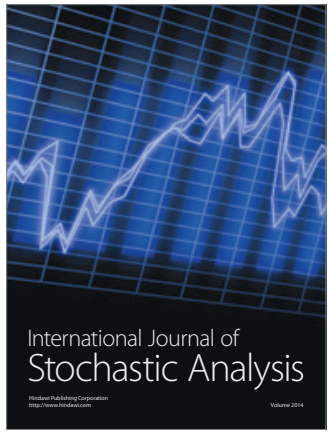

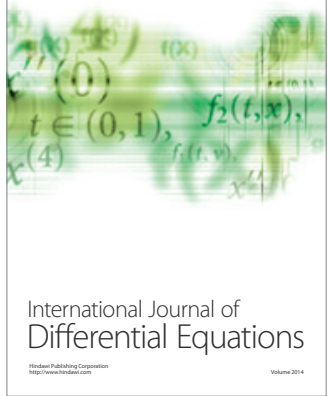
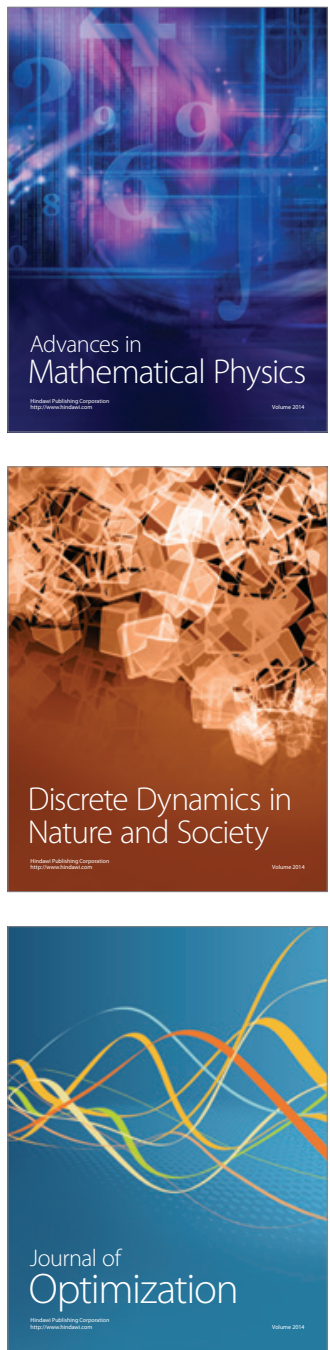